\title{
The Influences of Martensitic Transformations on Cavitation-Erosion Damage Initiation and Pitting Resistance of a Lean Austenitic Stainless Steel
}

\author{
Kai Yuan Wang ${ }^{a}$; Kin Ho Lo ${ }^{a, b *}$; Chi Tat Kwok ${ }^{a, b}$; Man Meng Wong ${ }^{a}$; In Wa Ionga ; Wenji Ai ${ }^{a}$ \\ a Department of Electromechanical Engineering, University of Macau, Macau \\ ${ }^{b}$ Institute of Applied Physics and Materials Engineering, University of Macau, Macau
}

Received: July 16, 2015; Revised: August 23, 2016; Accepted: September 07, 2016

\begin{abstract}
This paper looks into the influences of martensitic transformations on the cavitation-erosion (CE) damage initiation mechanism and pitting corrosion resistance of a lean austenitic stainless steel. The $\varepsilon$ and $\alpha^{\prime}$ martensites are prime sites of CE damage initiation for this steel, whereas grain boundaries are more favourable damage initiation sites for other similar steels. The profusion of $\varepsilon$ and $\alpha$ ' results in fast surface roughening during the $\mathrm{CE}$ process, which may cause a compromise in performance when this steel (or similar steels that have the same CE damage mechanism) is used for such applications as hydro-machinery or piping. $\varepsilon$ and $\alpha$ ' also detrimentally affect the pitting resistance of this steel and so they are expected to affect $\mathrm{CE}$ resistance adversely due to the synergism between $\mathrm{CE}$ and corrosion.
\end{abstract}

Keywords: Cavitation erosion; Lean austenitic stainless steel; Martensitic transformation; Pitting corrosion

\section{Introduction}

In applications involving high-speed flows, such as turbines and piping, cavitation erosion (CE) is always a problem. In piping systems, for instance, when there is a decrease in pipe diameter in a certain section, the local flow speed will increase and the fluid pressure will drop. If the pressure drop is sufficient, cavitation may occur and the inner wall of the pipe will experience erosion attacks ${ }^{1}$. The piping system may then suffer from vibration or the fluid conveyed in the pipe may experience an increase in pipe wall friction.

While carbon steels have been used in applications in which $C E$ is anticipated ${ }^{2}$, stainless steels are more preferred due to their higher corrosion resistance. In this regard, the martensitic grades ${ }^{3}$ and the precipitation-hardening grades ${ }^{3,4}$ of stainless steels have been considered useable. On the other hand, views on the austenitic grades appear to be varied. The CE resistance of austenitic stainless steels (AusSSs) has been stated to be good by some researchers ${ }^{3,4}$, but inferior by others ${ }^{5}$. Enhancement of CE resistance of the austenitic grades has been shown to be viable using surface engineering techniques, such as friction-stir welding ${ }^{6}$, laser treatments ${ }^{7}$, and nitriding 8 .

The lean AusSSs have been gaining popularity in recent years, as reflected by the continual development of new varieties. This popularity arises in a large part from their lower costs. Typically, these steels are high in Mn and N, but low in Ni which is expensive. The lean AusSSs (and the austenite phase of lean duplex stainless steels ${ }^{9}$ ) have a propensity for martensitic transformation, either through cooling ${ }^{10}$ or plastic deformation ${ }^{11}$. The transformation

* e-mail: KHLO@umac.mo routes may be from austenite $(\gamma)$ to the body-centred-cubic martensite $\left(\alpha^{\prime}\right)$ directly, or through the hexagonal-closepacked $\varepsilon$ martensite, or via deformation twins ${ }^{12}$.

When martensitic transformation occurs during the $\mathrm{CE}$ process, it provides a cushioning effect by taking up some of the impact energy from the collapsing bubbles. In this regard, martensitic transformation is considered beneficial, as it may reduce mass loss rate during the CE process ${ }^{13-15}$. Quite frequently, grain boundaries are reported as the prime initiation sites of CE damage ${ }^{13-15}$. The roles of $\varepsilon$ and $\alpha^{\prime}$ on CE damage initiation, on the other hand, appear to be less examined.

When a material undergoes $\mathrm{CE}$ in a corrosive medium, a synergism exists between $\mathrm{CE}$ and corrosion ${ }^{16}$, resulting in accelerated material attack. Because austenite and the $\alpha$ ' martensite may form a galvanic couple ${ }^{17}$, the $\alpha^{\prime}$ induced during the $\mathrm{CE}$ process is bound to detrimentally affect $\mathrm{CE}$ resistance.

The present work is not a chronicle of the CE process of a lean, high-Mn AusSS, as this has already been reported in depth for similar steels. Instead, this work looks into the influences of $\varepsilon$ and $\alpha$ ' martensites on CE damage initiation, damage morphology and pitting corrosion resistance of a Mn-containing AusSS in $3.5 \% \mathrm{NaCl}$, with the view of gaining a deeper understanding on the role played by $\varepsilon$ and $\alpha$ ' on the CE damage of lean AusSSs.

\section{Experimental Details}

The major elements present in the lean, high-Mn (lowNi) AusSS used in this work are shown in Table 1. The exact designation of the steel is unknown, however. This 
Table 1: Nominal composition of the lean steel used in this work (wt \%)

\begin{tabular}{lcccccccc}
\hline $\mathrm{Fe}$ & $\mathrm{Cr}$ & $\mathrm{Ni}$ & $\mathrm{Mn}$ & $\mathrm{S}$ & $\mathrm{Si}$ & $\mathrm{C}$ & $\mathrm{N}$ \\
\hline Balance & 14.67 & 1.05 & 10.77 & 0.05 & 0.79 & 0.12 & 0.26 \\
\hline
\end{tabular}

steel was chosen as it contained a relatively low Ni content (which is expensive) and a relatively high Mn content. Although the present steel is not a commercial grade with known designation, results reported below are believed to aid those making decisions on whether similar high-Mn, low-Ni AusSSs are chosen for applications for which CE is a concern.

All the samples for CE tests were solution-treated at $1100^{\circ} \mathrm{C}$ for $1 \mathrm{~h}$ and then then quenched rapidly into water. Vibratory CE tests were conducted using a sonicator according to ASTM G 32-06 at $25{ }^{\circ} \mathrm{C}$ in $3.5 \% \mathrm{NaCl}$. CE tests were interrupted at different times for observations of the surface damage morphology of samples using a Hitachi S3400N Type I scanning electron microscope (SEM). Potentiodynamic anodic polarisation tests were done in $3.5 \% \mathrm{NaCl}$ with a scan rate of $1 \mathrm{mV} / \mathrm{s}$ starting from $0.4 \mathrm{~V}$ under the open-circuit potential. For each condition, at least 3 polarisation tests were done. The polarisation curves presented in this work show the general trends of the polarisation tests. The samples for polarisation tests were variously solution-treated between $1100^{\circ} \mathrm{C}$ and $1300^{\circ} \mathrm{C}$ for $1 \mathrm{~h}$ and then water-quenched, with the view of assessing the effect of grain size on martensitic transformation and pitting corrosion resistance. Grain size measurements were done with a Leica Image Analyser (Model DMI3000M). The identification of $\varepsilon$ and $\alpha$ ' was done using X-ray diffractometry (XRD) with a Rigaku MiniFlex 600 diffractometer $(\mathrm{CuK} \alpha)$ with a scan rate of 0.1 degree/s.

\section{Results and Discussions}

\subsection{Influence of $\varepsilon$ and $\alpha$ 'on CE pit initiation and damage morphology}

According to XRD results, some as-solution-treated samples contained martensite, whereas some did not. The samples chosen for CE tests were those that did not contain any pre-existing martensite. The XRD spectra taken from one such originally martensite-free sample after it had gone through different $\mathrm{CE}$ times are shown in Figure 1. The variability in presence of martensite was chiefly due to the slight batch-to-batch variation in composition.

Before undergoing the CE process, the sample surface was flat and smooth (Figure 2). After undergoing the CE process for $15 \mathrm{~min}$, surface of the sample still looked relatively smooth under the SEM, but both $\varepsilon$ and $\alpha$ ' were already detectable (Figure 1(a) and (b)).

Under the SEM, traces of $\varepsilon$ and $\alpha^{\prime}$ could be seen to have formed in some regions. In other regions, these traces could only be vaguely discerned at high magnifications (Figure 3).
Very tiny CE pits could be seen to have formed. Although formation of these very tiny $\mathrm{CE}$ pits was not just restricted to the regions containing $\varepsilon$ and $\alpha$ ' traces, they were more numerous in these regions (see the inset of Figure 3).

One hour into the CE process, twin boundaries, grain boundaries, and martensite traces could be faintly seen, and all of these features appeared more or less at the same time (Figure 4). Diffraction peaks pertaining to $\varepsilon$ are more prominent than those pertaining to $\alpha$ ' throughout the $\mathrm{CE}$ process (Figure 1(b) and (c)). Nevertheless, diffractions peaks pertaining to $\alpha$ ' did exist throughout the CE process (Figure 1(d)).

In Figure 4, tiny CE pits may be seen to have formed on martensite traces ( $\varepsilon$ and $\left.\alpha^{\prime}\right)$. For the twin boundaries and grain boundaries, however, no noticeable CE pits had formed by this time. Therefore, although martensitic transformations are beneficial as far as mass loss rate is concerned ${ }^{13-15}, \varepsilon$ and $\alpha$ ' are the most favourable sites for CE damage initiation in this case. The relative importance of $\varepsilon$ and $\alpha$ ' for CE pit initiation, however, cannot be determined, as the two types of martensite are indistinguishable under the SEM. For this reason, $\varepsilon$ and $\alpha$ ' will be referred to collectively as martensite traces hereinafter.

Soon after the CE process began, numerous martensite traces had been produced in the sample. This profusion of martensite traces facilitated easy coalescence of the CE pits that had formed on them. For two CE pits that were close to each other, the small amount of material between them could be easily ploughed out, resulting in pit coalescence (Figure 5).

The higher susceptibility of martensite traces to CE damage initiation than twin and grain boundaries may be appreciated further by looking at the sample that had gone through the CE process for about 4 hours. In Figure 6, it is shown that the CE pits that had formed on twin boundaries were still in their infancy. Note that by this time, both twin and grain boundaries had been revealed more clearly. In Figure 6(b), the grain boundary above the two twin boundaries had not sustained too much damage.

Further down the CE process, more martensite traces were generated and on which new CE pits formed. For the martensite traces that had formed earlier, besides the formation of new $\mathrm{CE}$ pits, existing ones had grown along them, as indicated by the arrow in Figure 7(a)). By this time, $\mathrm{CE}$ damage had also grown along twin boundaries and grain boundaries. The extent of CE damage associated with these two types of boundaries, however, was less serious than that associated with the martensite traces. In Figure 7(a), the more severe CE damage associated with martensite traces 

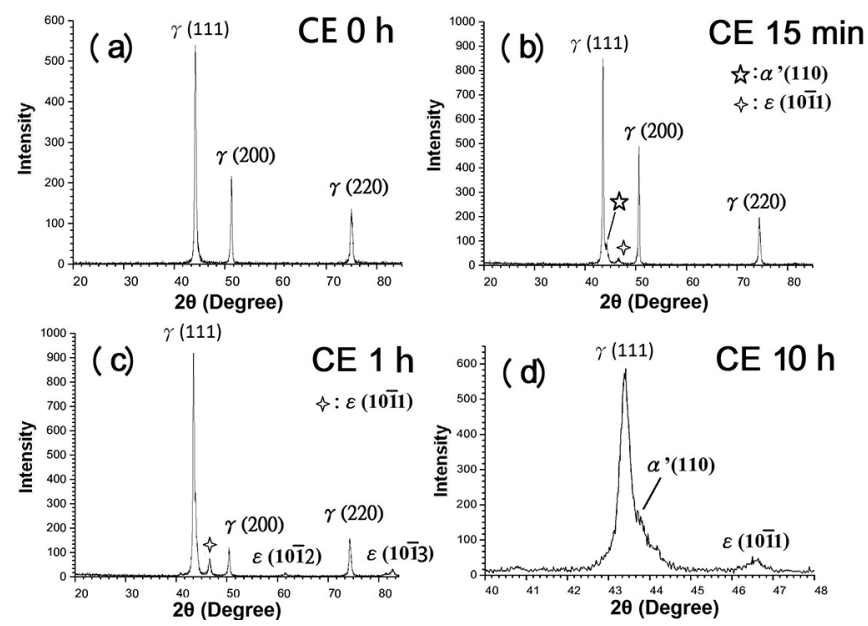

Figure 1: XRD spectra of an originally martensite-free sample that went through the CE process for different durations

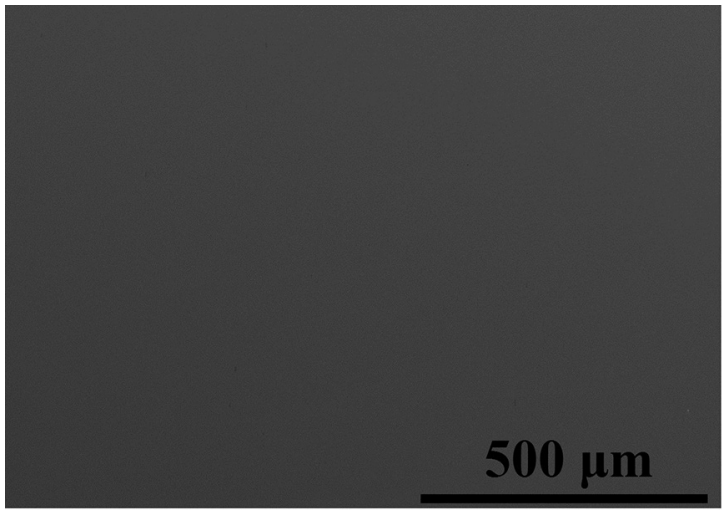

Figure 2: Appearance of a sample prior to undergoing the CE process.

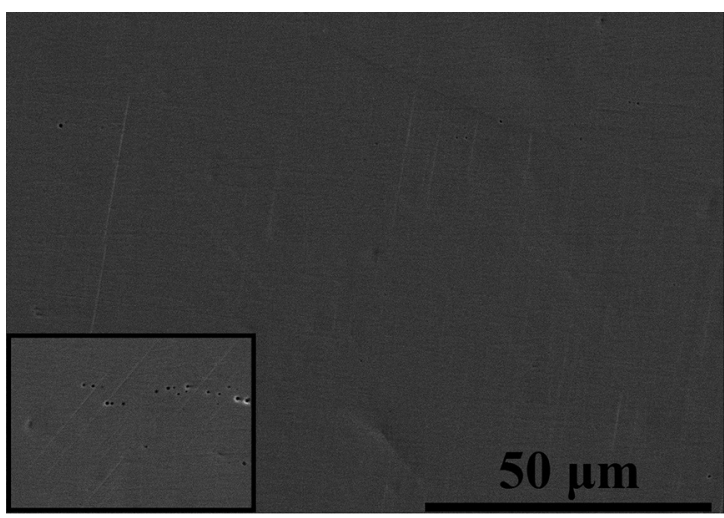

Figure 3: Appearance of a sample that had undergone the $\mathrm{CE}$ process for about $15 \mathrm{~min}$.

compared with the twin and grain boundaries is obvious. The CE damage associated with twin and grain boundaries in another region of the sample is demonstrated in Figure 7(b), and it may be seen that there was no significant dig-out of material along these boundaries.

As mentioned previously, innumerable martensite traces were produced during the CE process, which resulted in their

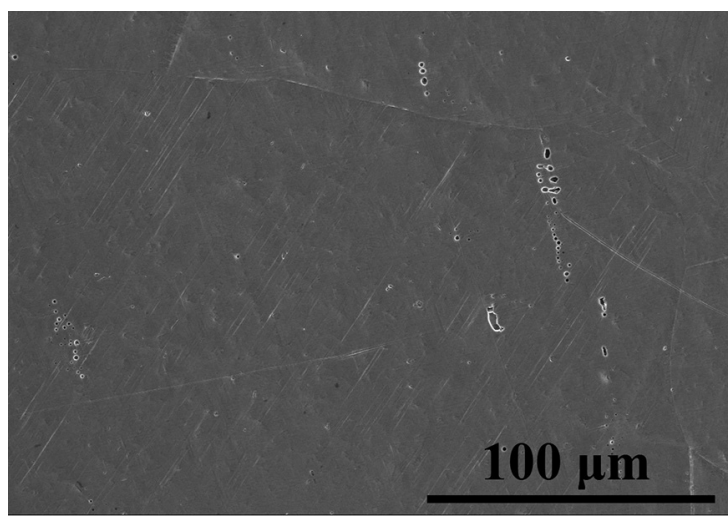

Figure 4: Formation of tiny CE pits on the martensite traces of a sample that had undergone the CE process for $1 \mathrm{~h}$.

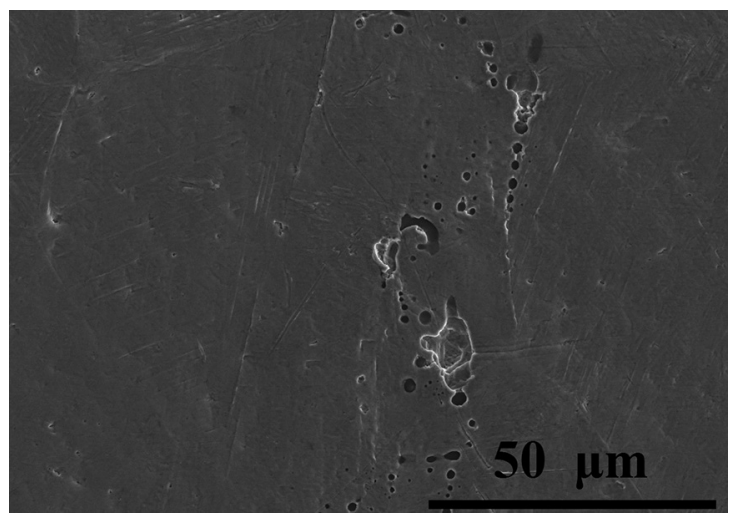

Figure 5: $\mathrm{CE}$ pits formed on martensite traces and their coalescence in a sample that had undergone the CE process for $3 \mathrm{~h} 15 \mathrm{~min}$.

close proximity to each other. Consequently, the CE pits that had formed on the martensite traces could join easily, thereby forming craters that propagated roughly perpendicularly to the martensite traces (Figure 8).

When two CE pits are near each other, the small bit of material between them could be easily torn off by the 

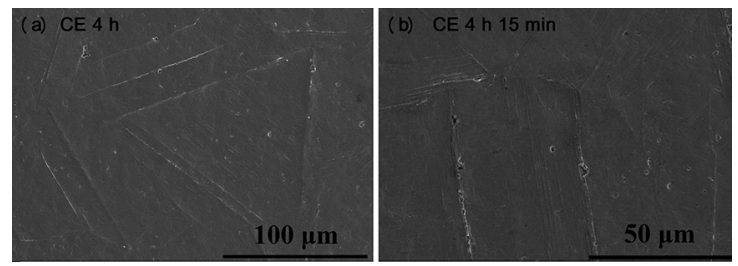

Figure 6: Formation of CE pits on twin boundaries of a sample that had gone through the CE process for (a) $4 \mathrm{~h}$ and (b) $4 \mathrm{~h} 15 \mathrm{~min}$.
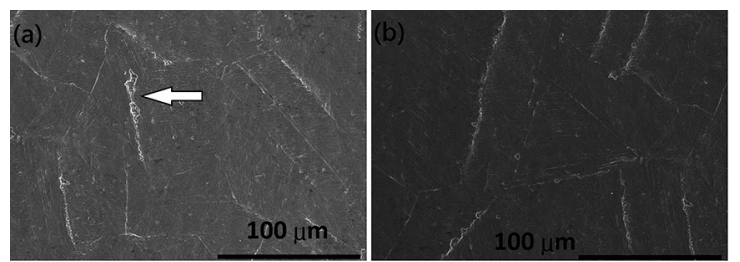

Figure 7: The surface morphology of a sample that had undergone the CE process for $16.5 \mathrm{~h}$.

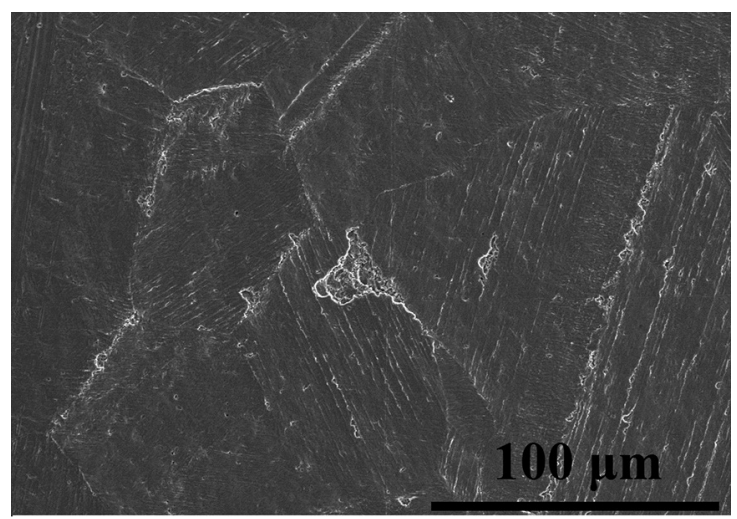

Figure 8: Growth of CE craters nearly perpendicular to martensite traces on a sample that had undergone the CE process for $18.5 \mathrm{~h}$.

shock waves generated by collapsing bubbles. Hence, the ductile peeling-off of material involved small bits and flakes, resulting in a damage morphology as shown in Figure 9. The main damage feature is wide-spread peeling-off of small and shallow bits of material. Once a small chunk of material is removed, $\varepsilon$ and $\alpha$ ' would form in the newly exposed material within a short time. And the above process will repeat itself. Eventually, a grainy damage morphology consisting of small globs would result (Figure 10). The grainy morphology shown in Figures 9 and 10 (a) is revealed more clearly in Figure 10(b), which is a close-up view of the region inside the rectangle in Figure 10(a). By this time, twin and grain boundaries had also sustained noticeable $\mathrm{CE}$ attacks.

In an Fe-16Cr-0.5C-3Mn AusSS, it was found using electron back-scattered diffraction (EBSD) that $\alpha^{\prime}$ had formed in its grain interiors during the $\mathrm{CE}$ process. But in the vicinity of grain boundaries, $\alpha^{\prime}$ was nearly absent ${ }^{14}$. The scarcity of $\alpha$ ' formation near the grain boundary regions in this steel resulted in damage initiation at its grain boundaries. The grain interiors of this steel, on the other hand, did not

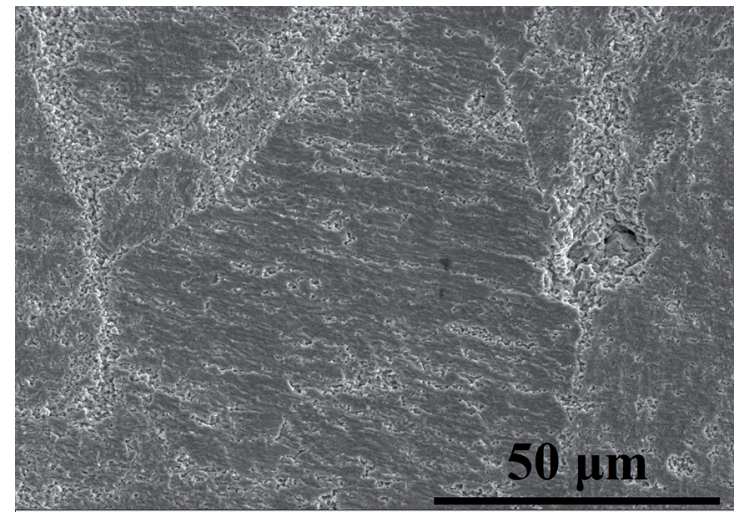

Figure 9: The surface of a sample that had undergone the $\mathrm{CE}$ process for about $30.5 \mathrm{~h}$.
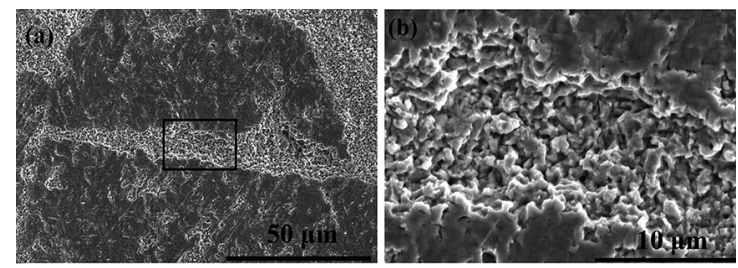

Figure 10: The grainy damage morphology of a sample that had undergone the CE process for $57 \mathrm{~h}$ (a) and a close-up view of the region inside the rectangle (b).

suffer from extensive CE damage because $\alpha$ ' formation dissipated some of the impact energy from the collapsing bubbles. It must be noted that the $\alpha$ ' of this steel did not appear to be damage initiation site. In the Fe- $16 \mathrm{Cr}-0.5 \mathrm{C}-3 \mathrm{Ni}$ AusSS investigated in the same work, extensive slip bands, instead of $\alpha^{\prime}$, had formed during the CE process ${ }^{14}$. These slip bands were found to be favourable damage initiation sites. The paucity of $\alpha$ ' formation in this steel was found to be responsible for its poorer $\mathrm{CE}$ resistance relative to its $\mathrm{Fe}-16 \mathrm{Cr}-0.5 \mathrm{C}-3 \mathrm{Mn}$ counterpart ${ }^{14}$. Similar results have also been observed in a series of Mn-containing AusSSs and Ni-containing AusSSs ${ }^{13}$.

For the lean steel used in this work, however, the situation seems to be a mix-up of those of the two steels mentioned in the preceding paragraph. For the present steel, $\varepsilon$ and $\alpha^{\prime}$, instead of slip bands, had formed and they extended all the way up to the grain boundaries. However, these two types of martensites seem to be favourable damage initiation sites, as opposed to the Fe-16Cr-0.5C-3Mn steel mentioned above. For the present lean steel, the formation of $\varepsilon$ and $\alpha$ ' almost everywhere led to fast roughening of its surface, which may cause the problems mentioned in Introduction when this steel (or similar steels) is used for hydromachinery or piping applications.

In some high interstitial CrMnCN AusSSs, twins and slip lines extending through to the grain boundaries were induced by the CE process ${ }^{18}$. Damage was observed to get initiated at grain boundaries, but not at the twins and slip 

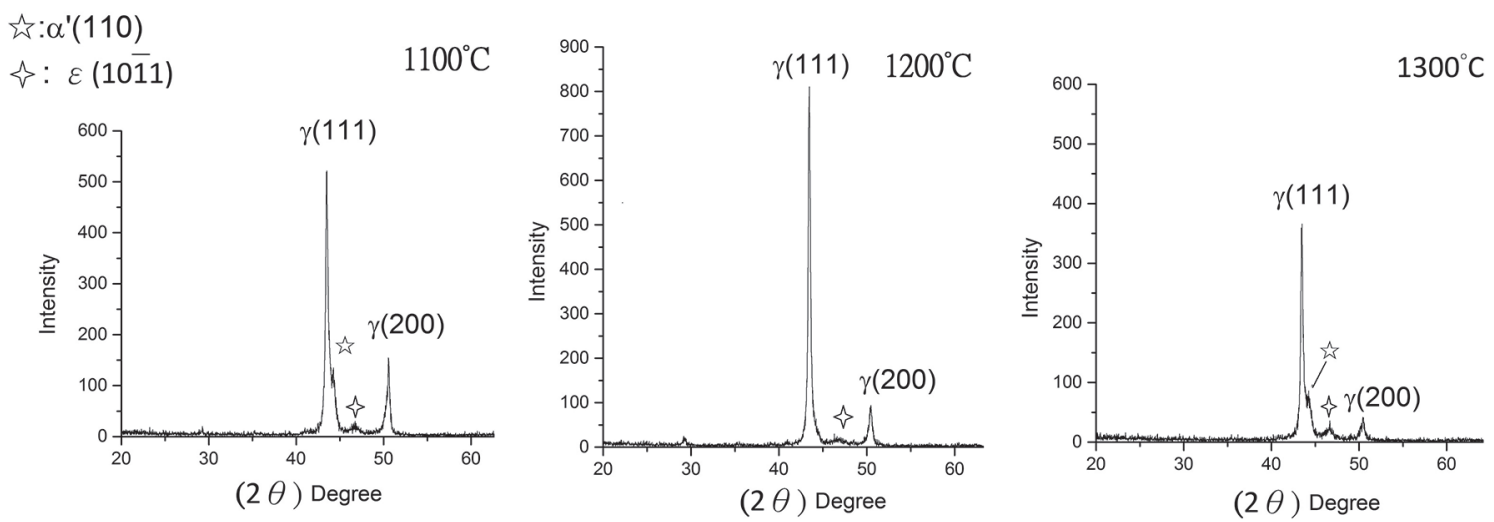

Figure 11: XRD spectra of the martensite-containing samples after water-quenching from different solution-treatment temperatures.

lines. For the present lean steel, damage appeared to get initiated at both the twin and grain boundaries almost at the same time. From the discussion above, it may be seen that there exist various mechanisms of CE damage initiation for these Mn-containing AusSSs.

Here, it must be emphasised that although $\varepsilon$ and $\alpha$ ' were found to be favourable initiation sites of CE damage for the present lean steel, it does not mean martensitic transformations are not beneficial to $\mathrm{CE}$ resistance. This is because the extent of surface damage may not directly correlate with mass loss rate. For example, for two AISI 304 AusSSs having different grain sizes, the one having a smaller grain size was found to have a lower mass loss rate during the CE process ${ }^{19}$. However, as far as the extent of surface damage is concerned, the AISI 304 steel having a small grain size was more severely attacked in comparison to the one having a bigger grain size, as grain boundaries were the main damage initiation sites ${ }^{19}$.

\subsection{Influence of $\varepsilon$ and $\alpha$ 'on pitting corrosion resistance}

Upon water-quenching between $1100^{\circ} \mathrm{C}$ and $1300{ }^{\circ} \mathrm{C}$, some of the samples contained $\alpha$ ' and $\varepsilon$ (Figure 11).

For these martensite-containing samples, their pitting potentials were reduced noticeably compared with their martensite-free counterparts that were quenched from the same temperature (Figure 12).

When the solution-treatment temperature was raised to $1300^{\circ} \mathrm{C}$ from $1100^{\circ} \mathrm{C}$, the pitting potential decreased progressively (Figure 13). The average grain size, however, increased markedly. An increase in grain size is detrimental to pitting corrosion resistance ${ }^{20}$. According to Ralston et al..$^{21}$, a metal that passivates in a given medium will be able to do so more easily when its grain size decreases, as an increase in surface area is conducive to passivation. This is certainly applicable to the present lean steel in $3.5 \% \mathrm{NaCl}$. An increase in grain size also facilitates martensitic transformation ${ }^{22}$,

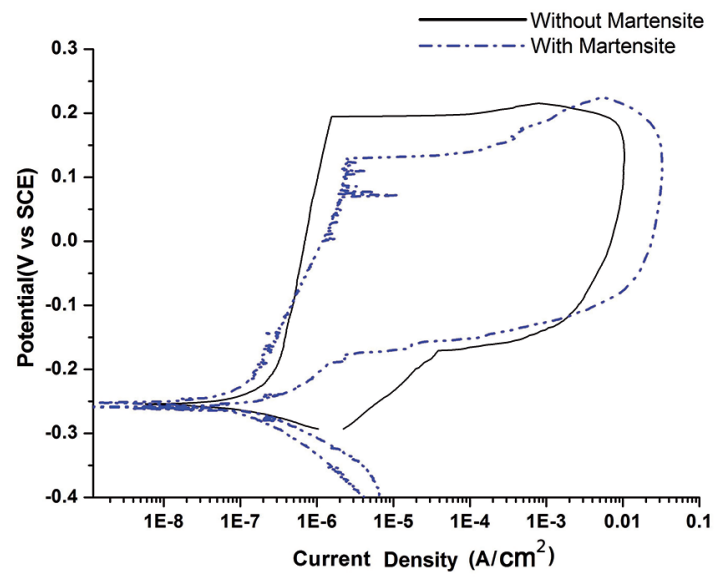

Figure 12: The influence of $\alpha$ ' and son the potentiodynamic anodic polarisation behaviour of the lean steel after quenching from $1100^{\circ} \mathrm{C}$.

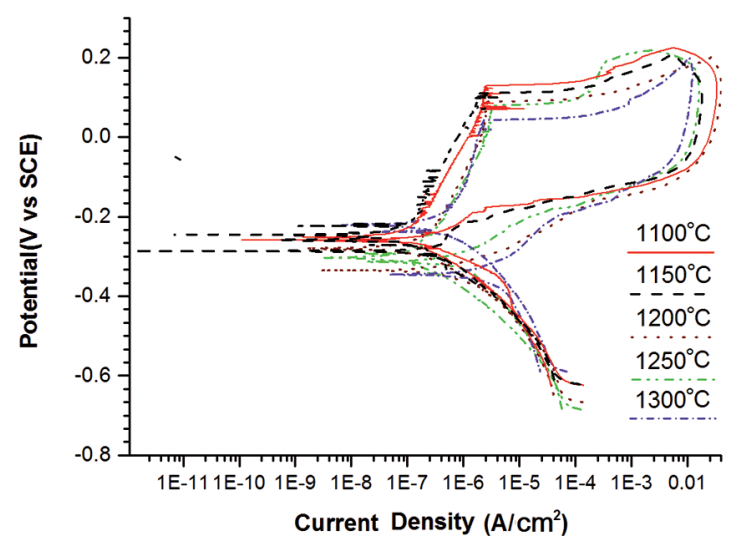

Figure 13: Change in potentiodynamic anodic polarisation behaviour of the lean steel with solution-treatment temperature.

${ }^{23}$. Both factors are responsible for the reduction in pitting potential as depicted in Figure 13.

Because corrosion and CE may act synergistically ${ }^{16}$, the reduction of pitting corrosion resistance by $\alpha^{\prime}$ and $\varepsilon$ is expected to compromise CE resistance in corrosive media. 


\section{Conclusions}

Martensitic transformations involvinge and $\alpha$ ' have been proved to be beneficial for cavitation erosion (CE) resistance of austenitic stainless steels by many researchers. However, $\varepsilon$ and $\alpha$ ' have been found to be the prime damage initiation sites for the present lean steel and they lead to fast surface damage and roughening. The omnipresence of $\varepsilon$ and $\alpha$ ' in the samples facilitates easy CE pit coalescence and so results in a grainy surface damage morphology. Additionally, $\varepsilon$ and $\alpha$ ' detract from the pitting corrosion resistance of this steel, which in turn is deleterious to $\mathrm{CE}$ resistance in corrosive media.

\section{Acknowledgements}

The present work was financially supported by Fundo para o Desenvolvimento das Ciências e da Tecnologia (FDCT) (Grant Nos.: 069/2012/A3).

\section{References}

1. Nagaya Y, Nurase M, Mizuyama S, Hattori S. Evaluation of incipient cavitation erosion for pipe wall at downstream of an orifice. In: Proceedings of the $7^{\text {th }}$ International Symposium on Cavitation; 2009 Aug 16-20; Ann Arbor, Michigan, USA. p. 1-5.

2. Ferreño D, Álvarez JA, Ruiz E, Méndez D, Rodríguez L, Hernández D. Failure analysis of a Pelton turbine manufactured in soft martensitic stainless steel casting. Engineering Failure Analysis. 2011;18(1):256-270.

3. Garverick L. Corrosion in the Petrochemical Industry. Materials Park: ASM International; 1994.

4. Khatak HS, Raj B, eds. Corrosion of Austenitic Stainless Steels: Mechanism, Mitigation and Monitoring. Cambridge: Woodhead Publishing; 2002.

5. Hajian M, Abdollah-zadeh A, Rezaei-Nejad SS, Assadi H, Hadavi SMM, Chung $\mathrm{K}$, et al. Improvement in cavitation erosion resistance of AISI 316L stainless steel by friction stir processing. Applied Surface Science. 2014;308:184-192.

6. Chiu KY, Cheng FT, Man HC. Laser cladding of austenitic stainless steel using NiTi strips for resisting cavitation erosion. Materials Science and Engineering: A 2005;402(1-2):126-134.

7. Sun GF, Zhang YK, Zhang MK, Zhou R, Wang K, Liu CS, et al. Microstructure and corrosion characteristics of 304 stainless steel laser-alloyed with $\mathrm{Cr}-\mathrm{CrB}_{2}$. Applied Surface Science. 2014;295:94-107.

8. dos Santos JF, Garzón CM, Tschiptschin AP. Improvement of the cavitation erosion resistance of an AISI 304L austenitic stainless steel by high temperature gas nitriding. Materials Science and Engineering: A. 2004;382(1-2):378-386.
9. Tavares SSM, Pardal JM, da Silva MR, de Oliveira CAS. Martensitic transformation induced by cold deformation of lean duplex stainless steel UNS S32304. Materials Research. 2014;17(2):381-385.

10. King HW, Larbalestier DC. Austenitic stainless steels at cryogenic temperatures: The compositional dependence of the $\mathrm{M}_{\mathrm{s}}$. Cryogenics. 1981;21(9):521-524.

11. Tavares SSM, Pardal JM, Gomes da Silva MJ, Abreu HFG, Silva MR. Deformation induced martensitic transformation in a 201 modified austenitic stainless steel. Materials Characterization. 2009;60(8):907-911.

12. Das A, Sivaprasad S, Chakranorti PC, Tarafder S. Morphologies and characteristics of deformation induced martensite during low cycle fatigue behaviour of austenitic stainless steel. Materials Science and Engineering: A. 2011;528(27):7909-7914.

13. Park MC, Kim KN, Shin GS, Yun JY, Shin MH, Kim SJ. Effects of $\mathrm{Ni}$ and $\mathrm{Mn}$ on the Cavitation Erosion Resistance of $\mathrm{Fe}-\mathrm{Cr}-\mathrm{C}-\mathrm{Ni} /$ Mn Austenitic Alloys. Tribology Letters. 2013;52(3):477-484.

14. Park MC, Shin GS, Yun JY, Heo JH, Kim DI, Kim SJ. Damage mechanism of cavitation erosion in austenite $\rightarrow$ martensite phase transformable Fe-Cr-C-Mn/Ni alloys. Wear. 2014;310(1-2):27-32.

15. Park MC, Kim KN, Shin GS, Kim SJ. Effects of strain induced martensitic transformation on the cavitation erosion resistance and incubation time of $\mathrm{Fe}-\mathrm{Cr}-\mathrm{Ni}-\mathrm{C}$ alloys. Wear. 2012;274275:28-33.

16. Kwok CT, Cheng FT, Man HC. Synergistic effect of cavitation erosion and corrosion of various engineering alloys in 3.5\% $\mathrm{NaCl}$ solution. Materials Science and Engineering: A. 2000;290(1-2):145-154.

17. Xu C, Hu G. Effect of deformation-induced martensite on the pit propagation behavior of 304 stainless steel. Anti-Corrosion Methods and Materials. 2004;51(6):381-388.

18. Niederhofer P, Huth S. Cavitation erosion resistance of high interstitial CrMnCN austenitic stainless steel. Wear. 2013;301(12):457-466.

19. Bregliozzi G, Di Schino A, Ahmed SIU, Kenny JM, Haefke H. Cavitation wear behaviour of austenitic stainless steels with different grain sizes. Wear. 2005;258(1-4):503-510.

20. di Schino A, Barteri M, Kenny JM. Effects of grain size on the properties of a low nickel austenitic stainless steel. Journal of Materials Science. 2003;38(23):4725-4733.

21. Ralston KD, Birbilis N, Davies CHJ. Revealing the relationship between grain size and corrosion rate of metals. Scripta Materialia. 2010;63(12):1201-1204.

22. Takaki S, Fukunaga K, Syarif J, Tsuchiyama T. Effect of grain refinement on thermal stability of metastable austenitic steel. Materials Transactions. 2004;45(7):2245-2251.

23. Yang HS, Bhadeshia HKDH. Austenite grain size and the martensitestart temperature. Scripta Materialia. 2009;60(7):493-495. 